\title{
An Algorithm for Foreground-Background Separation in Low Quality Patrimonial Document Images
}

\author{
Carlos A.B. Mello \\ Department of Computing Systems, University of Pernambuco \\ Recife, Brazil, 50720-001 \\ carlosadsc.upe.br
}

\begin{abstract}
In this article, we present a new algorithm to deal with foregroundbackground separation in very degraded documents. In particular, our work is applied to patrimonial document images which suffer from several types of degradation as aging effects, noise, back-to-front ink interference, etc. Our main objective is to correctly classify ink and paper to allow an efficient segmentation of the image creating high quality monochromatic images. This makes easier the broadcast of these images through the Internet. The new algorithm is based on the classical Shannon definition of entropy and a generalization defined as Tsallis Entropy and it is compared to 19 well-known classical algorithms, including $\mathrm{Dj} V u$ algorithm. It achieved the best results by analyzing precision, recall, accuracy, specificity, PSNR and MSE.
\end{abstract}

Keywords: Document processing, Image thresholding, Entropy.

\section{Introduction}

This research is part of the DocHist Project [8][9][10] for image processing of historical documents which aims the preservation and broadcast of a file of thousands of patrimonial documents. Even more, it is important to improve the readability of the digital documents. The archive used in this paper is composed of more than 6,500 letters and documents which amounts more than 30,000 pages from the end of the nineteenth century onwards.

For preservation purposes, the documents are digitized in 200 dpi resolution in true color and stored in JPEG file format with $1 \%$ loss for better quality/space storage rate. Even in this format each image of a document reaches, in average, $400 \mathrm{~KB}$. In spite of the common use of broadband Internet access nowadays, the visualization of a bequest of thousand of files is not easy. Even in JPEG all the archive consumes Giga Bytes of space. The conversion of the digital images to bi-level comes as a possible solution to this problem.

There are several research efforts in the development of image thresholding or binarization techniques [11]. This is the first step in some image processing applications as optical character recognition (OCR). Threshold algorithms search for a cut-off value that separates object and background in an image. This value defines which colors belong to one or another class. In the case of images of documents these two 
classes are the paper (the background) and the ink (the foreground). A good threshold value for this application is one that preserves in the final bi-level image all the information content of the document. This is quite a simple task when one deals with recent documents where, in general, the paper is almost completely clear which is not the case of ancient documents. This type of documents is degraded by the presence of background artifacts. For these cases, image enhancement techniques could be used first to improve the visual appearance of the image for further thresholding.

Images of historical documents present some unique features that make a binarization process very difficult 1) some documents are written on both sides of the paper and the ink from one side passes to the other side, creating a back-to-front interference (also known as bleed-through effect); 2) some paper sheets are very consumed and the paper has darkened over the time (the show-through effect); 3) the last case presents the documents where the ink has faded so much that it has almost the same color as the paper. Examples of these classes of documents can be seen in Fig. 1.
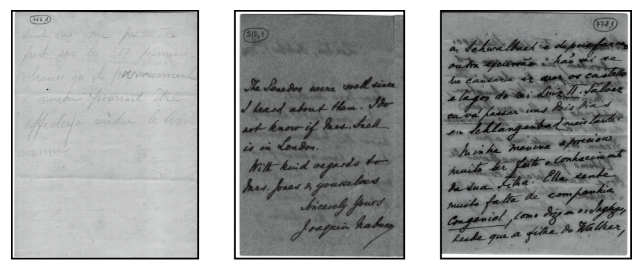

Fig. 1. Sample documents: (left) a very faded document; (center) a document with darkened paper and (right) a document with back-to-front interference

Next Section discusses some of the researches being developed for processing images of historical documents. The new proposed method is fully described in Section 3 and its results are disposed in Section 5, followed by the Conclusions of the paper.

\section{Image Thresholding of Patrimonial Documents}

Thresholding [11] is a classical problem for image processing. There is a great variety of algorithms defined for this purpose. Most of them are for general use, but there are specific algorithms for historical documents.

Previous works related to image processing of patrimonial documents can be found in literature. The problem of bleed-through interference is dealt in [16] where a canny edge detector is used to detect and to suppress undesired background patterns considering that the writing angle in the foreground opposes the writing angle in the background. This approach, however, does not deal with horizontal and vertical lines as can be found in a handwritten letter " $\mathrm{T}$ " for example. The same authors also propose a new method to deal with ink bleeding through the matching of the images from both sides of the paper which is a very difficult task [17].

The authors in [7] propose the use of multi-stage thresholding, i.e., different algorithms are used in different stages of the complete process in order to create the best image possible. The authors propose this and they also claim that global thresholding algorithms must not be used in this kind of images which is not validated in our work. 
It is proposed in [3] the use of quadtree decomposition to break down the image into sub-regions and to apply different thresholding algorithms in each of these regions. Background removal is also treated in [6] and [1].

An algorithm for background normalization is proposed in [15] to decrease the background influence and for further binarization. Unfortunately, the method is adjusted only for documents written on just one side of the paper.

A combination of global and local thresholding algorithms is presented in [5] using Iterative Global Thresholding (IGT). Sub-areas $n$ by $n$ of the image are analyzed to verify if they have more black pixels than they should have. The authors, however, do not explain how the size of the sub-areas must be defined.

Several well-known thresholding algorithms were tested in the images of our archive. None of them achieved satisfactory results. The tested algorithms are: Brink, C-Means, Fisher, Huang, Iterative Selection, Kapur, Kittler, Li-Lee, Mean Grey Level, Otsu, Percentage of Black, Pun, Renyi, Two Peaks, Wu-Lu, Yager and YeDanielsson.. A review of these methods can be found in [13] and the results of the application of some of them are presented in Fig. 2.
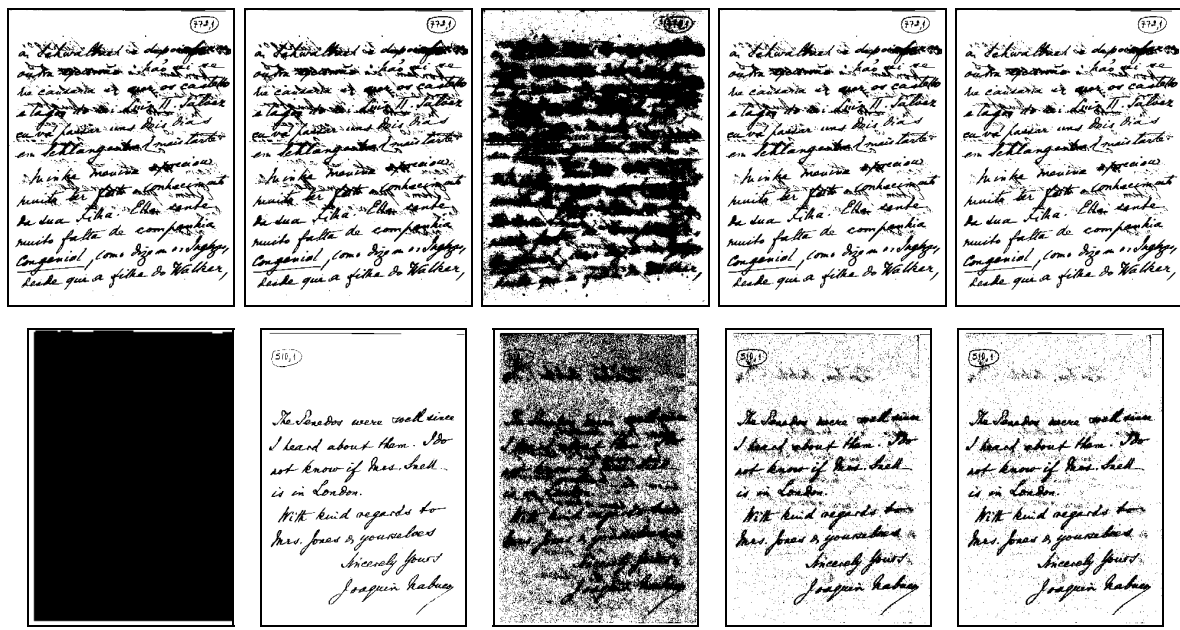

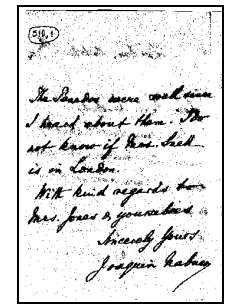

Fig. 2. Application of Brink, Huang, Pun, Percentage of Black and Otsu thresholding algorithms in (top) document of Figure 1-right and (bottom) document of Figure 1-center

\section{A New Tsallis-Entropy Based Thresholding Algorithm}

Tsallis entropy [18] has been considered a new information measure. It has been used in several image processing applications as Content Based Image Retrieval (CBIR) [12] and even thresholding [19][20] (however both papers present just the possibilities of the use of Tsallis entropy; certain parameters are not clearly specified in them). According to Tsallis, an universal definition of entropy is given by:

$$
H_{\alpha}(S)=\frac{1-\sum_{i} p(i)^{\alpha}}{\alpha-1}
$$


where $p(i)$ is a probability as in the classical definition of entropy and $\alpha$ is a real parameter. When $\alpha$ tends to 1 , Tsallis entropy reduces to Boltzmann-Gibbs entropy:

$$
H(S)=-\sum_{i} p(i) \ln (p(i))
$$

Shannon's definition of entropy $(H)[14]$ defines that:

$$
H(S)=-\sum_{i=0}^{n} p\left[s_{i}\right] \log \left(p\left[s_{i}\right]\right)
$$

Eq. 2 settles that if a system can be decomposed into two statistical independent subsystems, say $A$ and $B$, then $H$ has the extensive or additivity property. This means that $H(A+B)=H(A)+H(B)$. This fact is used in Pun's thresholding algorithm, for example. Tsallis entropy has a nonextensive property for statistical independent subsystems, defined by the following pseudo addivity entropic rule:

$$
H_{d}(A+B)=H_{d}(A)+H_{d}(B)+(1-\alpha) H_{d}(A) H_{d}(B)
$$

However, mathematically, Tsallis entropy (Eq. 1) can be broken into two parts:

$$
\begin{aligned}
& H_{\alpha}(S)=\frac{1-\sum_{i} p(i)^{\alpha}}{\alpha-1}=\frac{1}{\alpha-1}-\frac{1}{\alpha-1} \sum_{i} p(i)^{\alpha} \\
& H_{\alpha}(S)=\frac{X_{b}}{\alpha-1}+\frac{X_{w}}{\alpha-1}-\frac{1}{\alpha-1} \sum_{i=0}^{t} p(i)^{\alpha}-\frac{1}{\alpha-1} \sum_{i=t+1}^{255} p(i)^{\alpha} \\
& H_{\alpha}(S)=\left(\frac{X_{b}}{\alpha-1}-\frac{1}{\alpha-1} \sum_{i=0}^{t} p(i)^{\alpha}\right)+\left(\frac{X_{w}}{\alpha-1}-\frac{1}{\alpha-1} \sum_{i=t+1}^{255} p(i)^{\alpha}\right)
\end{aligned}
$$

where $X_{b}+X_{w}=1$. It can be defined then that:

$$
H_{\alpha}(S)=H_{b \alpha}(A)+H_{w \alpha}(B)
$$

with

$$
H_{b \alpha}(A)=\frac{X_{b}}{\alpha-1}-\frac{1}{\alpha-1} \sum_{i=0}^{t} p(i)^{\alpha}
$$

and

$$
H_{w \alpha}(B)=\frac{X_{w}}{\alpha-1}-\frac{1}{\alpha-1} \sum_{i=t+1}^{255} p(i)^{\alpha}
$$

In the equations above, $t$ is the threshold value. In our case, $t$ is the most frequent color in the image. It is reasonable to consider that this most frequent color is part of the background. $H_{b \alpha}$ is the entropy of the pixels below the color $t$ and $H_{w \alpha}$ is the entropy of the colors above the threshold $t$. The variable $t$ is also used to define the values of $X_{b}$ and $X_{w}$, as $X_{b}$ is the percentage of colors below $t$ and $X_{w}$ is the percentage of colors above $t$.

The $\alpha$ parameter is a real number and it characterizes the degree of nonextensivity. Its value is not fixed in Tsallis definition. For thresholding purposes, variations in this value can modify the cut-off value. For our project, $\alpha$ is equal to 0.3 for the most part of the images, changing in just one case as further explained. 
At first, the document images are separated into classes. There are three main classes of documents:

- Class 1: documents with few parts of text or documents where the ink has faded;

- Class 2: common documents with around $10 \%$ of text elements;

- Class 3: documents with more black elements than it should have; this includes documents with a black border or documents with bleed-through effect.

In order to classify an image as one of these classes, we evaluate Shannon entropy $(H)$ using Equation 2 but with the logarithmic basis taken as the product of the dimensions of the image. As defined in [4], changes in the logarithmic basis do not alter the definition of the entropy. The previous three classes of documents are defined by:

- $H \leq 0.26$ : Class 1 documents;

- $0.26<H<0.30$ : Class 2 documents;

- $H \geq 0.30$ : Class 3 documents.

These boundaries were defined in previous works [9][10] and they were adjusted in our new proposal. For example, the sample documents of Fig. 1 belong, from left to right, to classes $1(H=0.23), 2(H=0.29)$ and $3(H=0.32)$.

The entropy value can be broken into the entropy of black pixels, $H b$, and the entropy of the white pixels, $H w$, bounded by a threshold $t$ :

$$
\begin{aligned}
& H b=-\sum_{i=0}^{t} p\left[s_{i}\right] \log \left(p\left[s_{i}\right]\right) \\
& H w=-\sum_{i=t+1}^{255} p\left[s_{i}\right] \log \left(p\left[s_{i}\right]\right)
\end{aligned}
$$

In our case, $t$ is the most frequent color of the image.

For each of these classes, an analysis must be made to process the images that belong to them as can be seen next. The final threshold value, $t h$, is defined by:

$$
t h=m b * H_{b \alpha}+m w * H_{w \alpha}
$$

where $m b$ and $m w$ are multiplicative constants that are going to be defined for each class. $H_{b \alpha}$ and $H_{w \alpha}$ can be seen as projections of the $H \alpha$ value; changes in those values (generated by the product by $m w$ or $m b$ ) produces changes in $H \alpha$ itself.

\section{Class 1 Documents:}

As said before, this class involves documents with few ink elements or few text parts. This can happens in cases where the letter has just few words or the ink has faded. In this class, we can also find most part of the typewritten documents as, in general, the typewriter ink is not so strong as handwritten characters making them more susceptible to degradation of their colors.

Although the images of this class have similar features in some way, they differ in basic aspects as, for example, typewritten documents must occupy a complete sheet of paper (opposing the fact that this class groups documents with few text parts). Because of this, another aspect must be considered within this class. We must consider the distribution of the pixels of the original image using the values of $H w$ or $H b$. We choose $\mathrm{Hw}$ with no loss of generality. For these kind of images, we have: 
- If ( $H w \geq 0.1)$, then $m b=2.5$ and $m w=4.5$ (typewritten documents with dark ink and bright paper);

- If $(0.08<H w<0.1)$, then $m b=m w=6$ and $\alpha=0.35$ (documents with the ink faded);

- If $(H w \leq 0.8)$, then $m b=m w=4$ (documents with dark ink and paper).

\section{Class 2 Documents:}

The most common documents just need a boost in $H_{b \alpha}$ and $H_{w \alpha}$ to achieve the best threshold value. So, in general, the algorithm defines $m b=2.2$ and $m w=3$. Some darkened documents need another treatment. If a document belongs to class 2 and $\mathrm{HW}$ $>0.1$, then the value of mw decreases by half (i.e., $m w=1.5$ ), unless the most frequent color is greater than 200 (brighten documents) for which $m w=9$. Fig. 6 shows sample documents from class 2 darkened or not and their bi-level images.

\section{Class 3 Documents:}

These are the documents with more black pixels than expected in a normal document. In this class, we have documents with a black border or documents with back-to-front interference. As the ink from one side transposes to the other side, it creates an intermediary element in the image: there is no more just paper or background; the transposed ink is an element between them. In these cases, there is no need to increase the dark measures. The system must deal just with the paper and the transposed ink turning them to white. Because of this, the $m b$ parameter is fixed as 1 . In most documents, we have $m w=2$. Some cases, however, must be considered when the documents have brightened paper again. In this class, brighten paper documents are the ones with most frequent color $(t)$ greater than 185 :

- If $(t>=185)$ then

$$
\begin{aligned}
& \circ \quad \text { If }(0.071<h w<0.096) \text { then } m w=9 \text {; } \\
& \circ \quad \text { If }(0.096<=h w<0.2) \text { then } m w=6 \text {; }
\end{aligned}
$$

\section{Results}

The proposed algorithm was tested in a set of 200 images that are considered representative of the complete file. The results were considered very satisfactory by visual inspection. However a most objective measure is also necessary. In this set, $18 \%$ of the documents belong to the class $1,40 \%$ are from class 2 and $42 \%$ from class 3 .

To make a quantitative evaluation of the performance of the new algorithm, its results are compared against the ground truth knowledge (an ideal image with the background removed manually). This comparison is made using the concepts of: precision, recall, accuracy and specificity. In order to use a more automatic process, our analysis is based on the number of pixels correctly classified as paper or ink. For this purpose the ideal image is considered as what should be the final target of the algorithm. With this in mind, we can have the number of ink pixels correctly classified as ink (TP True Positives), the number of pixels correctly classified as paper (TN - True Negatives), the number of pixels misclassified as ink (FP - False Positive) and number of ink elements misclassified as paper (FN - False Negative), defining:

$$
\begin{array}{ll}
\text { Precision }=\mathrm{TP} /(\mathrm{TP}+\mathrm{FP}) & \text { Recall }=\mathrm{TP} /(\mathrm{TP}+\mathrm{FN}) \\
\text { Accuracy }=(\mathrm{TP}+\mathrm{TN}) /(\mathrm{TP}+\mathrm{TN}+\mathrm{FP}+\mathrm{FN}) & \text { Specificity }=\mathrm{TN} /(\mathrm{FP}+\mathrm{TN})
\end{array}
$$


Based on these measures, a good algorithm must have:

- Precision=1: there were no misclassification of the paper elements $(\mathrm{FP}=0)$;

- Recall=1: there were few mistakes in the classification of the ink elements $(\mathrm{FN}=0)$;

- Accuracy=1: there was no misclassification at all $(\mathrm{FP}+\mathrm{FN}=0)$;

- Specificity=1: every pixel that belongs to the paper were classified as that $(\mathrm{FP}=0)$.

Table 1 presents the average result for these four measures applied to a set of 200 documents binarized by the new proposed algorithm and classical algorithms in comparison with their ideal versions. Our algorithm achieved very good values for the four measures. We also analyzed the values of PSNR (Peak Signal-to-Noise Ratio) and MSE (Mean Square Error). Their average values are also presented in Table 1.

Table 1. Average values of precision, recall, accuracy, specificity, PSNR and MSE in a set of 200 bi-level documents generated by the new proposal and classical methods compared with their ideal version generated manually

\begin{tabular}{lllllll}
\hline Algorithm & Precision & Recall & Accuracy & Specificity & PSNR & MSE \\
\hline New Algorithm & $\mathbf{0 . 8 2}$ & $\mathbf{0 . 8 8}$ & $\mathbf{0 . 9 7}$ & $\mathbf{0 . 9 8}$ & $\mathbf{2 1 . 6 5}$ & $\mathbf{0 . 0 3}$ \\
Brink & 0.91 & 0.69 & 0.95 & 0.98 & 20,91 & 0.06 \\
C-Means & 0.88 & 0.79 & 0.93 & 0.99 & 15.61 & 0.27 \\
Fisher & 0.95 & 0.51 & 0.73 & 0.99 & 20.97 & 0.06 \\
Huang & 0.88 & 0.80 & 0.94 & 0.99 & 20.63 & 0.07 \\
Iterative Selection & 0.38 & 0.48 & 0.94 & 0.94 & 20.27 & 0.06 \\
Kapur & 0.88 & 0.79 & 0.93 & 0.98 & 20.30 & 0.05 \\
Kittler & 0.94 & 0.73 & 0.96 & 0.99 & 16.33 & 0.11 \\
Li-Lee & 0.00 & 0.57 & 0.89 & 0.89 & 20.13 & 0.04 \\
Mean Grey Level & 0.95 & 0.71 & 0.96 & 0.99 & 20.21 & 0.07 \\
Otsu & 0.81 & 0.81 & 0.97 & 0.98 & 21.18 & 0.03 \\
Percentage of Black & 0.99 & 0.23 & 0.63 & 0.99 & 19.20 & 0.05 \\
Pun & 0.94 & 0.69 & 0.93 & 0.99 & 10.41 & 0.37 \\
Renyi & 0.88 & 0.77 & 0.93 & 0.99 & 19.54 & 0.07 \\
Two Peaks & 0.87 & 0.82 & 0.95 & 0.98 & 8.49 & 0.62 \\
Wu-Lu & 0.94 & 0.71 & 0.95 & 0.99 & 18.89 & 0.06 \\
Yager & 0.99 & 0.17 & 0.39 & 0.91 & 21.37 & 0.05 \\
Ye-Denielsson & 0.87 & 0.77 & 0.93 & 0.99 & 19.86 & 0.05 \\
\hline
\end{tabular}

We should expect that the perfect algorithm must have the four measures next to 1 , high PSNR value and low MSE value. So a good algorithm must have all these features at the same time. Our new proposal has the higher PSNR and lower MSE. For precision, recall, accuracy and specificity, other algorithms achieved satisfactory results (as Otsu, Brink, Mean Grey Level, Huang) but our algorithm has a better performance in average.

Table 2 presents a second test as our algorithm is compared to images generated by $\mathrm{Dj} \mathrm{Vu}$ technology [2] which is defined specifically for document image thresholding and compression. Table 2 shows the average and standard deviation values of the 
same measures as before, comparing the images generated by our algorithm and the ones created by $\mathrm{Dj} V u$ and the ideal images.

Fig. 3 presents some very difficult images and the results of the application of the algorithm. In particular, Fig. 3-left presents the same document of Fig. 1-left. This is the best response ever achieved by an automatic algorithm for this image without any pre-processing technique for contrast enhancement.

Table 2. Average and standard deviation values of precision, recall, accuracy, specificity, PSNR and MSE in a set of 200 bi-level documents generated by the new proposal and $\mathrm{DjVu}$ technique in comparison with their ideal versions

\begin{tabular}{llll}
\hline Measure & & $\mathrm{DjVu}$ & New Algorithm \\
\hline \multirow{2}{*}{ Precision } & Average & 0.90 & 0.82 \\
& Standard Deviation & 0.12 & 0.12 \\
Recall & Average & 0.72 & 0.88 \\
& Standard Deviation & 0.24 & 0.09 \\
Accuracy & Average & 0.90 & 0.97 \\
& Standard Deviation & 0.20 & 0.01 \\
Specificity & Average & 0.99 & 0.98 \\
\multirow{2}{*}{ PSNR } & Standard Deviation & 0.01 & 0.02 \\
\multirow{2}{*}{ MSE } & Average & 19.60 & 21.65 \\
& Standard Deviation & 0.10 & 2.03 \\
& Average & 0.90 & 0.03 \\
\hline
\end{tabular}

Fig. 4 presents a document with differences of illumination along it. Even with this problem, our algorithm reached the best global threshold value possible as it can be seen in the comparison with classical well-known algorithms as Otsu and $\mathrm{Dj} V u$, which results are presented in the center part of this figure.

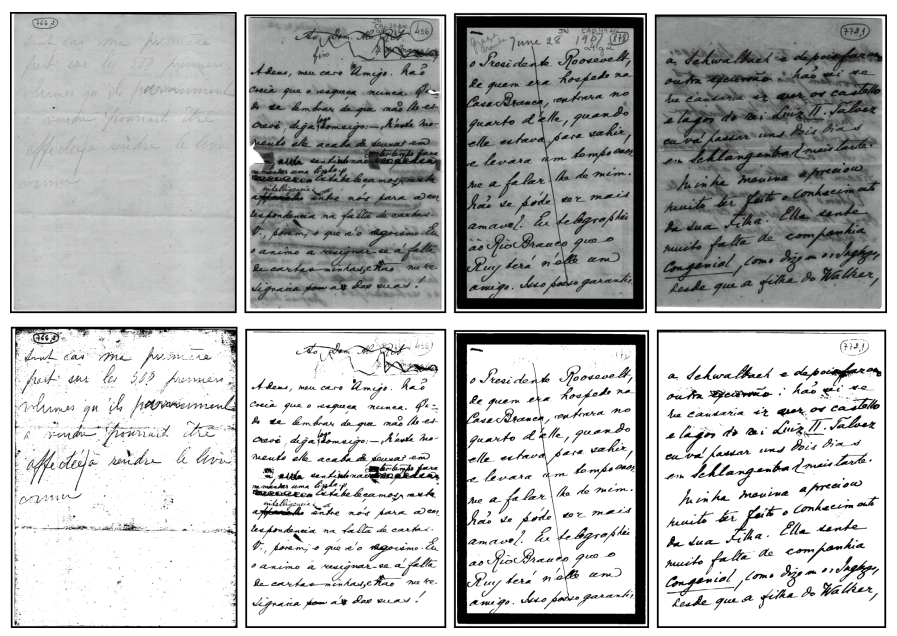

Fig. 3. (top) Sample documents and (bottom) their bi-level images produced by the new algorithm 
Other sample document from another database is shown in Fig. 5. This document is available at http://www.site.uottawa.ca/ edubois/documents. Fig. 5 presents a zooming into one of these documents and the binary versions generated by Otsu and our new algorithm. Again, our method achieved higher values of precision, recall, accuracy, specificity, PSNR and lower value of MSE. This shows that our method can be applied to other databases of similar features.
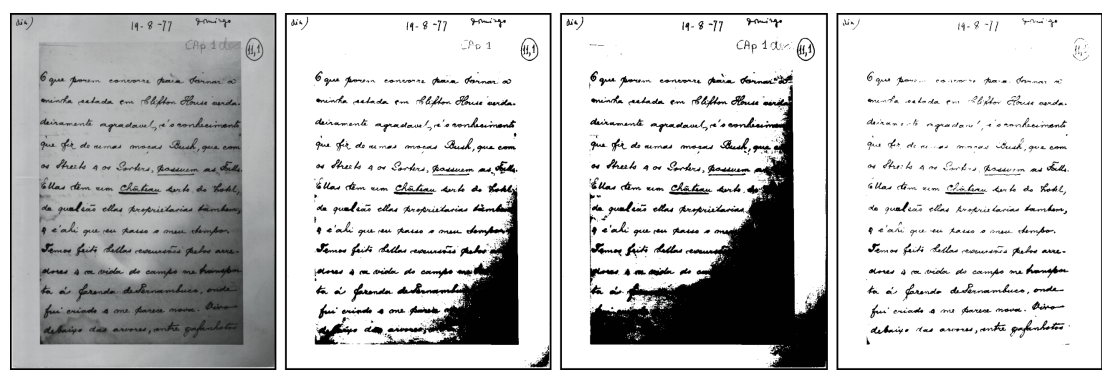

Fig. 4. (left) A document with different illumination along it, (right) the binarization produced by our new algorithm and at the center the results of the application of Otsu and $\mathrm{DjVu}$ algorithms

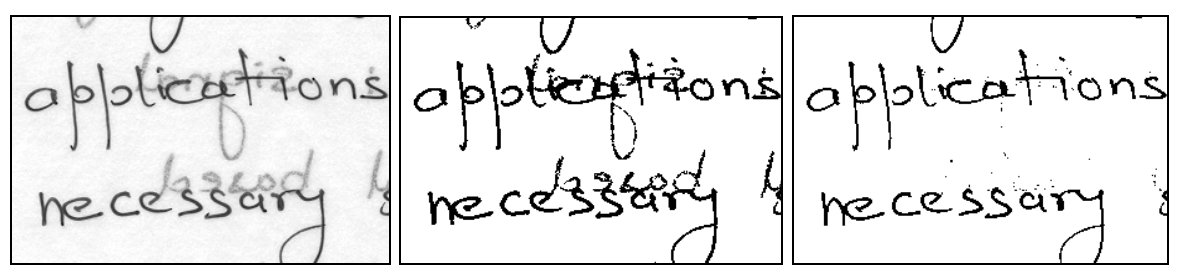

Fig. 5. (left) Zooming into another sample document from a different database; (center) bi-level image generated by Otsu algorithm and (right) the one produced by our new algorithm

\section{Conclusions}

This paper presents a new entropy-based thresholding algorithm for images of historical documents. The algorithm uses both Shannon and Tsallis definition of entropy to find the best cut-off value. The algorithm was applied in a set of 200 representative images of a file from the $19^{\text {th }}$ century and beginning of the $20^{\text {th }}$ century. The use of the algorithm was analyzed by visual inspection and by comparison with perfect bi-level images. The values of precision, recall, accuracy and specificity were evaluated for the complete set and the algorithm achieved satisfactory results.

Three classes of documents are identified using the classical Shannon entropy definition. After this, a set of rules is used to define the best threshold value. For this, Tsallis entropy is separated into two components which are boosted in order to define the cut-off value. The method proved to be very effective as could be analyzed using precision, recall, accuracy, specificity, PSNR and MSE metrics in comparison with several well-known thresholding algorithms, including the $\mathrm{Dj} V u$ technique. 


\section{References}

1. Antonacopoulos, A., Castilla, C.C.: Flexible Text Recovery from Degraded Typewritten Historical Documents. In: Int. Conf. on Pattern Recognition, pp. 1062-1065, Japan (2006)

2. Bottou, L., Haffner, P., Howard, P.G.: High Quality Document Image Compression with DjVu. Journal of Electronic Imaging, 410-425 (1998), http://www.djvu.org

3. Chen, Y., Leedham, G.: Decompose algorithm for thresholding degraded. Historical document images, Vision, Image and Signal Processing 152(6), 702-714 (2005)

4. Kapur, J.N.: Measures of Information and their Applications. J.Wiley \& Sons, Chichester (1994)

5. Kavallieratou, E., Stamatatos, E.: Improving the Quality of Degraded Document Images, Int. Conf. on Document Image Analysis for Libraries, pp. 340-349, France (2006)

6. Kennard, D.J., Barrett, W.A.: Separating Lines of Text in Free-Form Handwritten Historical Documents. In: Int. Conf. on Document Image Analysis for Libraries, pp. 12-23, France (2006)

7. Leedham, G., et al.: Separating Text and Background in Degraded Document Images - A Comparison of Global Thresholding Techniques for Multi-Stage Thresholding. In: International Workshop on Frontiers in Handwriting Recognition, pp. 244-249, Canada (2002)

8. Mello, C.A.B., et al.: Image Thresholding of Historical Documents: Application to the Joaquim Nabuco's File. In: Digital Cultural Heritage Conference - Eva Vienna, pp. 115122, Vienna, Austria (2006)

9. Mello, C.A.B.: Image Segmentation of Historical Documents: Using a Quality Index. In: International Conference on Image Analysis and Recognition, pp. 209-216, Portugal (2004)

10. Mello, C.A.B., et al.: Image Segmentation of Historical Documents. Visual (2000), Mexico (2000)

11. Parker, J.R.: Algorithms for Image Processing and Computer Vision. John Wiley \& Sons, Chichester (1997)

12. Rodrigues, P.S., et al.: Using Tsallis Entropy into a Bayesian Network for CBIR. In: Int. Conf. on Image Processing, pp. 1028-1031, Genova (2005)

13. Sezgin, M., et al.: Survey over image thresholding techniques and quantitative performance evaluation. Journal of Electronic Imaging, vol. 13(1) (2004)

14. Shannon, C.: A Mathematical Theory of Communication. Bell System Technology Journal 27, 370-423 (1948)

15. Shi, Z., Govindaraju, V.: Historical Document Image Enhancement Using Background Light Intensity Normalization. In: International Conference on Pattern Recognition, pp. 473-476, UK (2004)

16. Tan, C.L., et al.: Removal of Interfering Strokes in Double-Sided Document Images. In: Workshop on Applications of Computer Vision, pp. 16-21, USA (2000)

17. Tan, C.L., et al.: Restoration of Archival Documents Using a Wavelet Technique. IEEE Trans.on Pattern Analysis and Machine Intelligence 24(10), 1399-1404 (2002)

18. Tsallis, C.: Possible Generalization of Boltzmann-Gibbs statistics. Journal of Statistical Physics 52(1-2), 479-487 (1988)

19. Yan, L., et al.: An Application of Tsallis Entropy Minimum Difference on Image Segmentation, World Congress on Intelligent Control and Automation, pp. 9557-9561, China (2006)

20. Yan, L., et al.: Image Segmentation based on Tsallis-entropy and Renyi entropy and Their Comparison. In: Int. Conf. on Industrial Informatics, pp. 943-948, Singapore (2006) 